\title{
RIN Transfer Mitigation Technique Using Broadband Incoherent Pump in Distributed Raman Amplified Transmission Systems
}

\author{
Md Asif Iqbal ${ }^{1}$, Mingming Tan ${ }^{1}$, and Paul Harper ${ }^{1}$ \\ ${ }^{l}$ Aston Institute of Photonic Technologies, Aston University, Aston Triangle, Birmingham, UK \\ Tel: (0121) 204 3512,e-mail:m.tan1@aston.ac.uk
}

\begin{abstract}
The paper reviews the recent advances on RIN transfer mitigation techniques used in distributed Raman amplified long-haul coherent transmission systems. The use of a broadband first order pump in substantial pump to signal RIN transfer mitigation has been demonstrated experimentally in both bidirectional and backward only pumping schemes. The generation process of a novel broadband, low RIN and incoherent pump is also reported in detail. In a $10 \times 120 \mathrm{~Gb} / \mathrm{s}$ DP-QPSK WDM transmission system, our proposed pumping schemes shows maximum transmission reach up to $8332 \mathrm{~km}$ and significant reach extensions compared with conventional low RIN and narrowband Raman pump sources.
\end{abstract}

Keywords: Raman amplification, coherent fibre optic communications.

\section{INTRODUCTION}

Distributed Raman amplification (DRA) can effectively reduce the signal power evolution along the transmission fibre, and therefore lead to an enhancement in the transmission performance/capacity, thanks to the improved balance between the amplified spontaneous emission (ASE) and the Kerr nonlinearities [1,2]. The benefit of reduced signal power profile is significant, as it does not only improve the signal noise ratio (SNR) of the transmitted signal, but it also maximises the efficiency of nonlinearity compensation using mid-link optical phase conjugation (OPC) or nonlinear Fourier transform (NLFT) [3,4]. To minimise the signal power variation, forward-propagated (bi-directional) pumping schemes are required [5]. However, forward-propagated pumping would bring severe relative intensity noise (RIN) penalty to the signal, which limits the transmission performance in long-haul repeatered systems [6-9]. There have been a number of ways to mitigate the RINrelated penalty, but there are always limiting factors including the reduced pump-signal Raman gain efficiency or using higher order/ customised forward pumping [10-14]. Consequently, a RIN-penalty-free first order pump is preferred to maximise the Raman gain efficiency and enhance the transmission performance simultaneously. In this paper, we experimentally demonstrate the recent progress on the development of a novel first order broadband incoherent pump sources. Using such pump source can effectively mitigate the RIN penalty from both forward and backward directions, and extend the maximum reach up to $25 \%$ in long-haul transmission systems.

\section{THE PROPOSED BROADBAND INCOHERENT PUMP AS A FORWARD PUMP}

Figure 1(a)-(c) shows the schematic diagrams of distributed Raman amplification configurations. The Raman gain of all the configurations was to compensate the attenuation of the $83.32 \mathrm{~km} \mathrm{SSMF}$. In Fig. 1(a), the proposed first order broadband incoherent pump is illustrated. The overall pump generation can be seen as a dual stage process. In the first stage, $10 \mathrm{~km} \mathrm{SSMF}$ was used as the Raman gain medium, and the broadband Rayleigh backscattered light centred at $1453 \mathrm{~nm}$ was generated/amplified by the $1365 \mathrm{~nm}$ second order pump. The light was effectively amplified spontaneous emission (ASE) which was similar to a typical Raman gain spectrum [15]. In the second stage, the ASE light was fed into a $10 \mathrm{~km}$ TrueWave fibre and amplified by the residual $1366 \mathrm{~nm}$ pump from the first stage. The second stage acted as a discrete Raman amplifier to maximize the output power [16]. The final output power at $1453 \mathrm{~nm}$ could be up to $250 \mathrm{~mW}$ when using $3.8 \mathrm{~W}$ pump power at $1365 \mathrm{~nm}$, and the $3 \mathrm{~dB}$ bandwidth of this broadband pump was $\sim 12 \mathrm{~nm}$ [17]. The pump was used in the forward-propagated directions in Fig. 1(a), and the pump power was $\sim 50 \mathrm{~mW}$. Raman fibre laser based amplification was used near the output section, where the second order pump at $1365 \mathrm{~nm}$ and a $\sim 95 \%$ reflectivity FBG with a centre peak at $1455 \mathrm{~nm}$ and a $3 \mathrm{~dB}$ bandwidth of $\sim 0.5 \mathrm{~nm}$ was used. A "half-open" cavity random distributed feedback (random DFB) fibre laser was formed due to the Rayleigh backscattering from the fibre and the high reflectivity FBG located at the fibre output $[18,19]$. In Fig. 1(b), the low-RIN $(-134 \mathrm{~dB} / \mathrm{Hz})$ semiconductor laser with much narrower $3 \mathrm{~dB}$ bandwidth $(\sim 0.8 \mathrm{~nm})$ was used as the forward pump for comparison. As illustrated in Fig. 1(c), Raman fibre laser based amplification with backward pumping only was used as a baseline to evaluate the RIN-related impact when using different kinds of forward pumps.

Signal power profiles along the transmission fibre using different configurations were illustrated in Fig. 1(d). It can be seen that using forward pumping can effectively reduce the signal power variations which leads to an improvement in amplifier noise figure [5]. The RIN of the signals at the span output was demonstrated in Fig. 1(e), in which using forward broadband incoherent pumping shows the lowest, and using semiconductor laser as the forward pump shows the highest. The RIN from the broadband ASE pump was averaged out due to 
the non-degenerate four-wave mixing effect, as the phase was quickly varied over a large bandwidth of the pump [20], which led to the lowest RIN transfer to the signal. In order to evaluate the impact in long-haul repeatered transmission, a recirculating loop experiment using standard $10 \times 120 \mathrm{~Gb} / \mathrm{s}$ dual polarisation QPSK transmitter was conducted as shown in [6]. Figure 1(f) shows the measured Q factors versus signal launch power per channel for the $1545.32 \mathrm{~nm}$ signal at $3333 \mathrm{~km}$ (40 recirculations). The optimum launch powers for all the configurations stayed the same, as the signal power profiles were similar and therefore gave the similar trade-off between ASE noise and nonlinearity. The Q factor using the broadband incoherent pump was the highest, up to $12 \mathrm{~dB}$. This was because the reduction in signal power profile led to a slightly better balance in ASE and nonlinearity, and the signal RIN was suppressed thanks to the use of the broadband incoherent forward pump. Although the signal power variation was also reduced when using the low RIN semiconductor laser, the RIN transfer still occurred and consequently the Q factor improvement due to the improved signal power profile was cancelled due to the RIN-related penalty from the forward pump. Backward pumped random fibre laser configuration showed a slightly better $\mathrm{Q}$ factor in comparison with the semiconductor laser configuration, thanks to the absence of the forward-propagated pump. Figure $1(\mathrm{~g})$ shows the $\mathrm{Q}$ factors versus transmission distances for different configurations, in which the broadband forward pumping shows the longest maximum transmission distance of $8332 \mathrm{~km}$, up to $25 \%$ enhancement in reach.
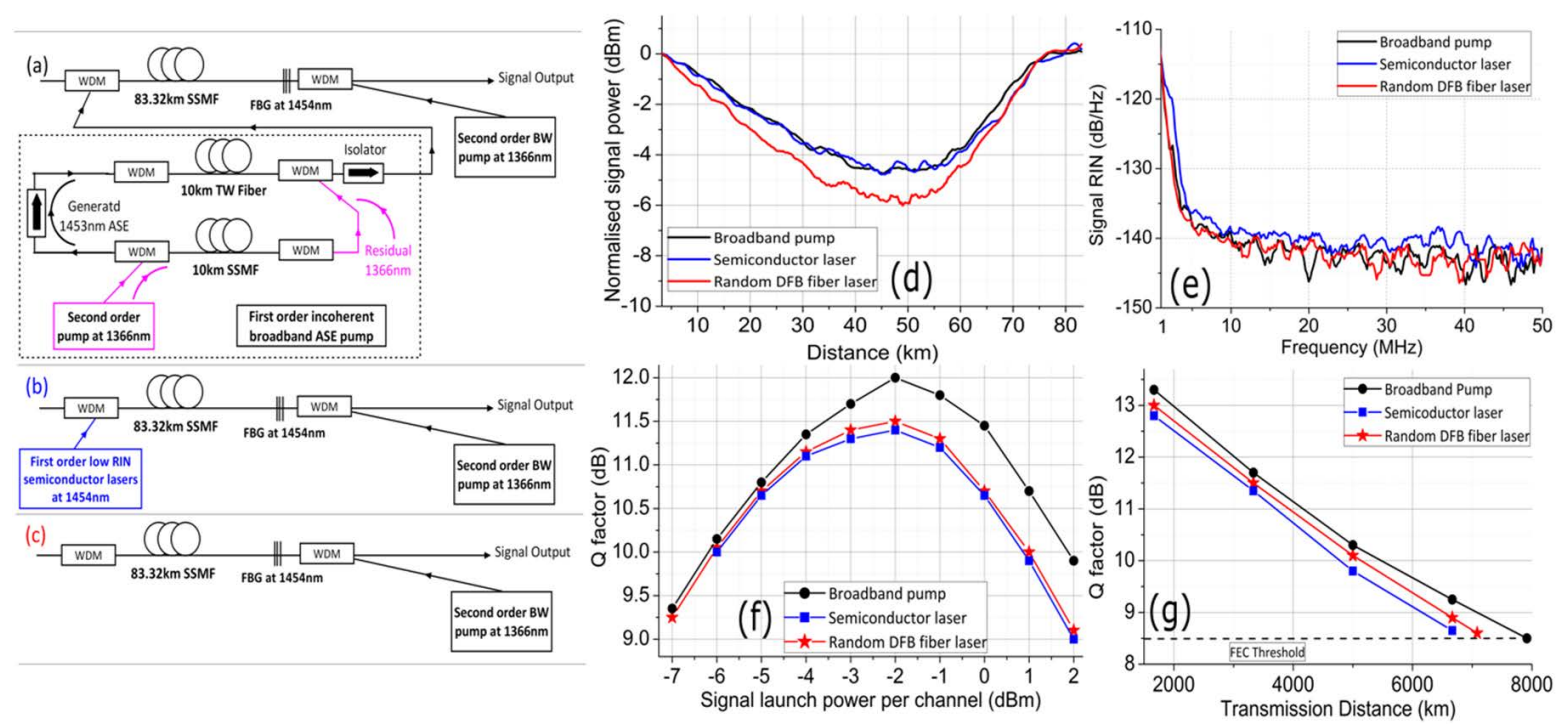

Figure 1. Raman fibre laser based amplification: (a) with first order broadband incoherent forward pump, (b) with first order low RIN semiconductor pump, (c) with backward pumping only; (d) Signal power profiles along the fibre; (e) RIN of the signal at the span output; $(f)$ Q factors versus signal launch power at $3333 \mathrm{~km}$ for the signal at $1545.32 \mathrm{~nm}$; $(\mathrm{g}) Q$ factors versus transmission distances.

\section{THE PROPOSED BROADBAND INCOHERENT PUMP AS A BACKWARD PUMP}

It was commonly believed that the RIN transfer rarely occur from the backward pump to the signal due to the averaging over long transmission fibre and low cut-off frequency of the RIN transfer function [9]. However, in long-haul repeatered transmission systems over a great number of spans, this very small amount RIN transfer can still impact the transmission performances.

Different backward pumping only configurations were illustrated in Fig. 2(a)-(c). Similarly, the overall Raman gain was to compensate only the fibre loss. In Fig. 2(a), dual order backward only pumping with the $20 \mathrm{~mW}$ broadband incoherent seed pump at $1453 \mathrm{~nm}$ was used. $20 \mathrm{~mW}$ low RIN semiconductor laser seed pump was replaced in Fig. 2(b). The random fibre laser based amplification with backward pumping only was shown in Fig. 2(c). As the purpose was to evaluate the RIN impact in long-haul transmission, the signal power profiles shown in Fig. 2(d) remained the same for all the Raman configurations and therefore the balance between ASE noise and nonlinearity also remained equal. The signal RIN at the span output was shown in Fig. 2(e). Signal RIN using the broadband incoherent seed clearly gave the lowest RIN, and the random DFB fibre laser configuration gave the highest RIN. This was because the random DFB fibre laser was effectively an oscillated laser cavity, and therefore there would be laser components propagated from both forward and backward directions in terms of the perspective of the signal $[6,17]$. Consequently, the RIN from the forward-propagated random laser would transfer the RIN to the signal. In addition, the RIN of the random fibre laser was relatively high $(\sim-115 \mathrm{~dB} / \mathrm{Hz})$ [16]. Compared with the broadband incoherent seed pump, the use of low RIN semiconductor laser as a backward seed pump still transferred its RIN to the signal at some extent. Figure 2(f) demonstrates the $\mathrm{Q}$ factors versus signal launch power per channel using the three different schemes at $3333 \mathrm{~km}$. 
As expected from the signal power profiles, the optimum signal launch power remained the same for all the schemes. However, due to the differences in RIN transfer to the signal, using the broadband incoherent seed pump gave the lowest signal RIN, and therefore showed the best $\mathrm{Q}$ factor up to $11.7 \mathrm{~dB}$. Using the semiconductor laser seed pump showed a slight decrease in Q factor, and using random fibre laser configuration gave the worst $\mathrm{Q}$ factor. This showed that even using the conventional low RIN semiconductor laser pump in the backward-propagated direction still showed a $\mathrm{Q}$ factor penalty in long-haul repeatered transmission systems. As illustrated in Fig. 2(g), the enhancement in maximum reach using the proposed broadband incoherent seed pump was $833 \mathrm{~km}(12 \%)$, compared with the other two configurations.
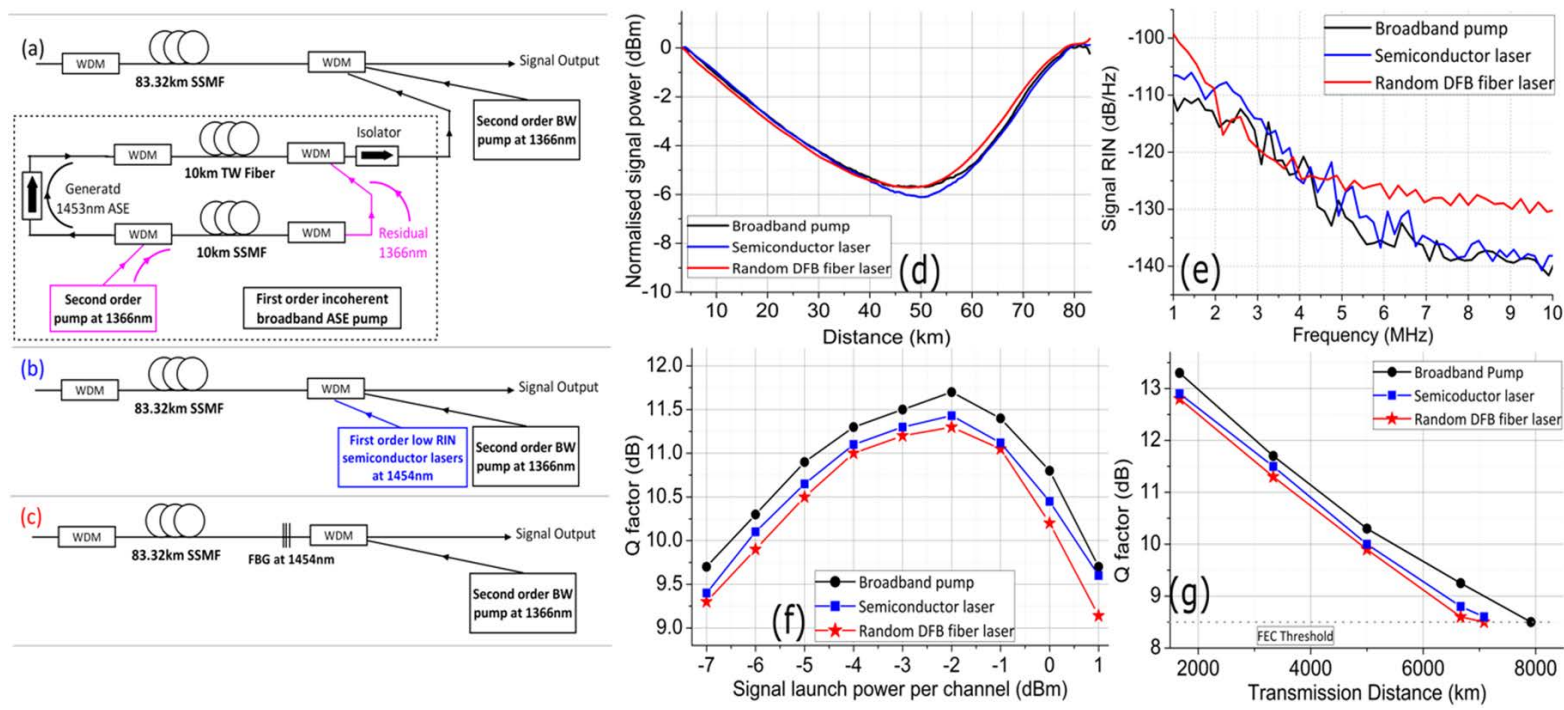

Figure 2: (a) Dual order backward pumping with first order broadband incoherent seed pump; (b) Dual order backward pumping with first order low RIN semiconductor seed pump; (c) Raman fibre laser based amplification with backward pumping only; (d) Signal power profiles along the fibre; (e) RIN of the signal at the span output; (f) Q factors versus signal launch power at $3333 \mathrm{~km}$ for the signal at $1545.32 \mathrm{~nm}$; (g) Q factors versus transmission distances.

\section{CONCLUSIONS}

In conclusion, we demonstrated a novel broadband incoherent ASE pump which can be used to mitigate the RIN transfer and allow the maximum reach enhancement in long-haul repeatered transmission systems. In DRA with bidirectional pumping, the improvement in reach using the proposed pump can be up to $25 \%$. In backward pumped DRA, although the RIN transfer from the backward-propagated pump was less significant, using the proposed broadband pump can still improve the maximum transmission distance by $12 \%$, in comparison with conventional low RIN semiconductor laser or FBG based random fibre laser based amplification. Such broadband pump source has the potential of providing a flatter gain and reducing the number of pumps in broadband transmission systems.

\section{ACKNOWLEDGEMENTS}

The work was funded by FP7 ITN programme ICONE (No. 608099), UK EPSRC programme grant UNLOC (EP/J017582/1) and PEACE (EP/L000091/1). We thank Dr Zhongyuan. Sun, Changle Wang, and Prof. Lin Zhang for providing the FBGs. Original data for this work is available through Aston Research Explorer (http://doi.org/xxxxx/researchdata.aston.ac.uk.xxxxx).

\section{REFERENCES}

[1] W. S. Pelouch, "Raman amplification: An enabling technology for long-haul coherent transmission systems," J. Lightwave Technol., vol. 34, no. 1, pp. 6-19, Jan. 2016.

[2] L. Galdino et al., "Amplification schemes and multi-channel DBP for unrepeatered transmission," J. Lightwave Technol, vol. 34, no. 9, pp. 2221-2227, May 2016.

[3] P. Rosa et al., "Signal power asymmetry optimisation for optical phase conjugation using Raman amplification," Opt. Express, vol. 23, pp. 31772-31778, 2015.

[4] J. E. Prilepsky et al., "Nonlinear inverse synthesis and eigenvalue division multiplexing in optical fiber channels," Phys. Rev. Lett., vol. 113, no. 1, p. 013901, 2014.

[5] J. -C. Bouteiller et al., "Quasi-constant signal power transmission," in Proc. ECOC, 2002. 
[6] M. Tan et al., "Evaluation of 100G DP-QPSK long-haul transmission performance using second order copumped Raman laser based amplification,” Opt. Express, vol. 23, pp. 22181-22189, 2015.

[7] L. Xu et al., "Experimental verification of relative phase noise in Raman amplified coherent optical communication system," J. Lightwave Technol., vol. 34, no. 16, pp. 3711-3716, 2016.

[8] S. Burtsev et al., "Pump-to-signal cross-polarization scattering in coherent dual-polarized systems with forward Raman amplification," in Proc. OFC, Anaheim, CA, USA, 2016, paper Th2A.48.

[9] C. R. S. Fludger et al., "Pump to signal RIN transfer in Raman fiber amplifiers," J. Lightwave Technol., vol. 19, no. 8, pp. 1140-1148, 2001.

[10] M. Tan et al., "Transmission performance improvement using random DFB fiber laser based Raman amplification and bidirectional second-order pumping," Opt. Express, vol. 24, no. 3, pp. 2215-2221, 2016.

[11] M. Morimoto et al., "Co-propagating dual-order distributed Raman amplifier utilizing incoherent pumping," IEEE Photon. Technol. Lett., vol. 29, no. 7, pp. 567-570, 2017.

[12] M. Tan et al., "RIN mitigation and transmission performance enhancement with forward broadband pump," IEEE Photon. Technol. Lett, vol. 30, no. 3, pp. 254-257, Feb. 2018.

[13] G. Rizzelli et al., "Impact of input FBG reflectivity and forward pump power on RIN transfer in ultralong Raman laser amplifiers," Opt. Express, vol. 24, no. 25, pp. 29170-29175, 2016.

[14] F. Gallazzi et al., "Performance optimization in ultra-long Raman laser amplified 10×30 GBaud DP-QPSK transmission: Balancing RIN and ASE noise," Opt. Express, vol. 25, 21454-21459 (2017).

[15] D. V. Churkin et al., "Raman fiber lasers with a random distributed feedback based on Rayleigh scattering," Physical Review A, vol. 82, no. 3, pp. 033828, 2010.

[16] M. A. Iqbal et al., "Enhanced transmission performance using backward-propagated broadband ASE pump," IEEE Photonics Technology Letters, vol. 30, no. 9, pp. 865-868, May 2018.

[17] M. A. Iqbal et al., "On the mitigation of RIN transfer and transmission performance improvement in bidirectional distributed Raman amplifiers," Journal of Lightwave Technology, vol. 36, no. 13, pp. 2611-2618, Jul. 2018.

[18] S. B. Papernyi et al., "Third-order cascaded Raman amplification," in Proc. OFC, 2002, paper FB4.

[19] W. L. Zhang et al., "Low threshold 2nd-order random lasing of a fiber laser with a half-opened cavity," Opt. Express, vol. 20, no.13, pp. 14400- 14405, 2012.

[20] K. Keita et al., "Relative intensity noise transfer of large-bandwidth pump lasers in Raman fiber amplifiers," J. Opt. Soc. Am. B., vol. 23, no. 12, pp. 2479-2485, 2006. 